\title{
Carotid Doppler as a Screening Tool for Silent Cerebral Stroke in Hypertensive Patients
}

\author{
Hesham M. Farouk, Osama T. Galal, Nada N. Ali
}

\begin{abstract}
Department of radiology, Benha faculty of medicine, Banha University, Egypt.
\end{abstract}

Correspondence to: Nada N. Ali, Department of radiology, Benha faculty of medicine, Banha University, Egypt

Email:

nadasalamaomar@yahoo.com

Received: 31 August 2021

Accepted: 5 December 2021

\begin{abstract}
Background: The concept of 'silent stroke' or 'asymptomatic cerebrovascular lesion' has been established by many investigations, and prevalence, risk factors, clinical outcome and associated pathological changes (WMCs and underlying vasculopathy) have been studied. The aim of the work is to use Carotid Doppler as a screening tool for silent cerebral stroke with MRI imaging in hypertensive patients. Methods: This study included 50 hypertensive patients. All patients underwent magnetic resonance imaging of the brain searching for Asymptomatic cerebral infarction. Patients with silent brain stroke underwent Duplex sonography of carotid artery. Results: Normal MRI findings occurred in only $8 \%$ of patients. Lacunar infarction, ischemic changes and microbleeds occurred in $78 \%, 72 \%$ and $24 \%$ of patients. High PSV occurred in $48 \%$ of the studied patients and $90 \%$ of them had normal EDV. Normal carotid findings occurred in
\end{abstract} only $10 \%$ of patients. Atherosclerosis without plaques were detected in $30 \%$ of patients while $60 \%$ of patients had combined atherosclerosis with plaques, Abnormal carotid Doppler findings detect 43 out of 46 patients with findings suggestive of silent stroke with sensitivity $93.5 \%$ and normal carotid Doppler occurred in 2 out of 4 patients with normal MRI study with specificity $50 \%$. Positive predictive value (PPV) was $95.6 \%$ while negative predictive value (NPV) was $40 \%$ and accuracy $90 \%$. There is significant agreement between presence of abnormal findings on carotid Doppler and findings of silent stroke by MRI, Conclusion: Carotid atherosclerosis can be proposed as a marker for susceptibility to ischemic cerebral damage.

Keywords: Carotid; Doppler; cerebral; Stroke; hypertension 


\section{Introduction}

Hypertension is the most important modifiable vascular risk factor for stroke and it is commonly widespread in the aging population. For a long time, treatment for hypertension has been focused on blood pressure (BP) levels as the main measure to determine the need and type of treatment. This approach has changed over the past years, emphasizing that diagnosis and treatment should be based on the quantification of global cardiovascular risk. According to the European guidelines on hypertension, common variables used to stratify risk are based on the presence of vascular risk factors (family history of premature cardiovascular disease, smoking habits, glucose and lipid parameters) plus further identification of clinical or subclinical target organ damage. Hypertension-related disease in several organs might indicate progression and markedly increase the risk beyond that caused by the BP levels or risk factors presence. Since evidences advise for different goals of treatment in high risk individuals as compared with lower risk hypertensive, whenever possible, it is recommended to measure target organ damage in different tissues (1).
Regarding brain as hypertension target organ, subclinical or "silent" vascular brain lesions (i.e. infarcts, microbleeds, white matter changes) are often detected by neuroimaging in asymptomatic patients. Specifically, silent brain infarcts are five times more frequent than stroke in general population, and increase significantly both with advancing age and hypertension (2).

Carotid artery stiffness, measured with sonography, is an independent risk factor for cerebrovascular disease, cognitive impairment, and mortality. Distal embolization, arising from degenerative breakdown or thrombotic occlusion of complex plaques, are important mechanisms of stroke in patients with atherosclerotic internal carotid artery stenosis. (3)

Carotid ultrasound is a good screening modality for imaging the carotid bifurcation and measuring blood velocities. The main purpose to diagnose carotid artery disease is prevention of stroke in a high risk population. (4).

The aim of the work is to use Carotid Doppler as a screening tool for silent cerebral Stroke with MRI imaging in hypertensive patients 


\section{Patients and methods}

This cross sectional study included 50 hypertensive patients. All the patients were enrolled from Radiology Department at Damietta Specialized Hospital and Mansoura University Hospital, during the period from June 2020 to May 2021. After approval of the ethics committee of researchers of Benha University and obtaining written consent from all patients.

\section{Inclusion criteria:}

1. Either sex.

2. Age 50-70 years.

3. Hypertensive patients.

\section{Exclusion criteria:}

1. Patients below 50 years or above 70 years.

2. Patients with cerebral hemorrhage or old stroke.

3. Comatosed patients.

4. Patients presenting with other concomitant neurologic or psychiatrics disease.

\section{Data procession:}

- Throughfull clinical history taking and previous available examination

- Radiological examination.

\section{Techniques:}

All patients underwent magnetic resonance imaging of the brain searching for asymptomatic cerebral infarction. The FLAIR images are more sensitive to ischemic change than any of the other conventional pulse sequences. Diffusionweighted and ADC MR imaging allow the delineation of the actual ischemic lesion with high sensitivity and specificity. Patients with silent brain stroke underwent Duplex sonography of carotid artery as it is currently the principal and, undoubtedly, the most accurate noninvasive and inexpensive diagnostic technique available for the evaluation of internal carotid artery stenosis. It provides information about the presence and severity of carotid stenosis, the velocity and characteristics of blood flow, and plaque morphology.

\section{Neuro-radiological Examination Used in} This Study:

All the patients then suspected to routine magnetic resonance Imaging (MRI) using standard head coils, in neutral supine position. The patients were asked to avoid deep breathing during examination. Routine conventional MRI (cranial axial and sagittal T1-weighted images, T2 weighted image, fluid attenuation inversion recovery 
(FLAIR), Diffusion weighted images and apparent diffusion coefficient) was acquired.

DWI scans and the apparent diffusion coefficient maps were used to detect silent ischemic lesions. The lesion was defined as a focal hyperintense area detected by the fluid attenuated inversion recovery sequence, corresponding to a restricted diffusion signal in the DWI sequence and confirmed by apparent diffusion coefficient mapping to rule out shine through artifacts. The higher level of diffusion sensitization was replicated in each of the 3 principal gradient directions ( $\mathrm{x}, \mathrm{y}$, and $\mathrm{z}$ planes). The diffusion-weighted images from each of the 3 diffusion-sensitized acquisitions were separately displayed for analysis. Besides DWI, other sequences, which comprised the axial T1-weighted image, the T2-weighted image and the FLAIR image were included. On each scan, the number and distribution (cortical, subcortical, or deep white matter) of lesions were recorded.

\section{Apparatus:}

They were examined using 1.5 T MRI and LOGIQ P5 and P7 ultrasound systems with 7.5 MHZ linear probe, no special preparation for the examination.

\section{Statistical analysis}

Analysis of data was done by IBM computer using SPSS (statistical program for social science version 20) as follows: Description of quantitative variables as mean, SD and range, Description of qualitative variables as number and percentage, Chi-Square test was used to compare qualitative variables, Unpaired t-test was used to compare quantitative variables, in parametric data ( $\mathrm{SD}<50 \%$ mean ), Mann Whitney test was used instead of unpaired t-test in nonparametric data, Paired t-test was used to compare quantitative variables in the same group before and after, $\mathrm{P}$ value $>0.05$ insignificant. $\mathrm{P}<0.05$ significant. $\mathrm{P}<0.01$ highly significant.

\section{Results}

All the studied patients were hypertensives, $40 \%$ of them were males. Age ranged from 50 to 74 years with mean 60.72 years, table 1

Normal MRI findings occurred in only $8 \%$ of patients. Lacunar infarction, ischemic changes and microbleeds occurred in $78 \%$, $72 \%$ and $24 \%$ of patients. (Figure 1)

High PSV occurred in $48 \%$ of the studied patients and $90 \%$ of them had normal EDV, figure 2 
Normal carotid findings occurred in only $10 \%$ of patients. Atherosclerosis without plaques was detected in $30 \%$ of patients while $60 \%$ of patients had combined atherosclerosis with plaques, table 2 .

There is non-significant association between carotid Doppler findings and each of MRI findings including lacunar infarction, ischemic changes, microbleeds or normal findings among the studied patients. Among four patients with normal MRI findings, two had atherosclerosis, while two had normal carotid Doppler findings. Thirty nine patients with lacunar infarction, 11 had atherosclerosis, 26 patients had combined atherosclerosis and plaques and 2 had normal Doppler study. All patients with microbleeds had atherosclerosis either alone in five patients or with plaques in the remaining six patients. Thirty six patients with ischemic changes, out of them, 11 had atherosclerosis, 23 had combined atherosclerosis and plaques and two had normal Doppler findings. (Table 3)

There is statistically non-significant association between EDV and findings on carotid Doppler among the studied patients. All patients with normal carotid findings had normal EDV. (Table 4)
There is statistically significant association between PSV and findings on carotid Doppler among the studied patients. All patients with normal carotid findings had normal PSV. (Table 5)

Abnormal carotid Doppler findings detect 43 out of 46 patients with findings suggestive of silent stroke with sensitivity 93.5\% and normal carotid Doppler occurred in 2 out of 4 patients with normal MRI study with specificity $50 \%$. Positive predictive value (PPV) was $95.6 \%$ while negative predictive value (NPV) was $40 \%$ and accuracy $90 \%$. There is significant agreement between presence of abnormal findings on carotid Doppler and findings of silent stroke by MRI. (Table 6)

Abnormal carotid Doppler findings detect all patients with findings with high EDV with sensitivity $100 \%$ and normal carotid Doppler occurred in 5 out of 45 patients with normal EDV study with specificity $11.1 \%$. Positive predictive value (PPV) was $11.1 \%$ while negative predictive value (NPV) was $100 \%$ and accuracy $20 \%$. There is non-significant slight agreement between presence of abnormal findings on carotid Doppler and high EDV (Table 7) 


\section{Cases}

Case No 1: female patient, 51 years old, hypertensive for 11 years. MRI brain: Few foci of abnormal SI in both occipital regions display low signal in T1WI, high signal in T2WI and FLAIR images. Old lacunar infarcts (Figure 3), B-Mode: Evidence of bilateral diffuse atherosclerotic changes in the extracranial carotid artery walls in the form of thickening of the intima-media complex $1.2 \mathrm{~mm}$. An adherent atheromatous plaque at the right common carotid artery. It is echogenic, homogenous, having regular surface measure $12 \times 2 \mathrm{~mm}$. Spectral analysis: figure 4
- PSV was $68 \mathrm{~cm} / \mathrm{sec}$

- EDV was $18 \mathrm{~cm} / \mathrm{sec}$

- Stenosis ratio $=27 \%$

Case No 2: female patient, 53 years old, hypertensive for 8 years. MRI brain: Multiple periventricular lacunar infarctions at both cerebral hemispheres in $\mathrm{T} 2$ and FLAIR axial images. (Figure 5), B-Mode: Evidence of bilateral diffuse atherosclerotic changes in the extracranial carotid artery walls in the form of thickening of the intima-media complex $1.1 \mathrm{~mm}$. Spectral analysis: figure 6

- Elevated PSV reaching $121 \mathrm{~cm} / \mathrm{sec}$.

- $\mathrm{EDV}=29 \mathrm{~cm} / \mathrm{sec}$

Table (1) Distribution of the studied patients according to demographic data:

\begin{tabular}{lll}
\hline & $\mathbf{N}=\mathbf{5 0}$ & \% \\
\hline Gender: & 30 & 60 \\
Female & 20 & 40 \\
Male & & \\
Age (year): & $60.72 \pm 6.25$ & \\
Mean \pm SD & $50-74$ & 100 \\
Range & 50 & Hypertension: \\
Yes & &
\end{tabular}

Table (2) Distribution of the studied patients according to carotid doppler findings

\begin{tabular}{lll}
\hline Carotid Doppler & $\mathbf{N = 5 0}$ & \% \\
\hline Atherosclerosis & 15 & 30 \\
Atherosclerosis with Plaque & 30 & 60 \\
Normal & & \\
& 5 & 10 \\
Carotid doppler: & & \\
Abnormal & 45 & 90 \\
Normal & 5 & 10 \\
\hline
\end{tabular}


Table (3) Relation between findings on MRI and carotid doppler among the studied patients:

\begin{tabular}{llllll}
\hline MRI & Atherosclerosis & $\begin{array}{l}\text { Carotid Doppler } \\
\text { Atherosclerosis with } \\
\text { plaques }\end{array}$ & Normal & $\chi^{\mathbf{2}}$ & $\mathbf{p}$ \\
& $\mathbf{N = 1 5 ( \% )}$ & $\mathbf{N = 3 0 ( \% )}$ & $\mathbf{N = 5 ( \% )}$ & & \\
\hline Lacunar infarction & $11(78.6)$ & $26(83.9)$ & $2(40)$ & MC & 0.113 \\
Ischemic changes & $11(78.6)$ & $23(74.2)$ & $2(40)$ & MC & 0.282 \\
Microbleeds & $5(35.7)$ & $6(19.4)$ & $0(0)$ & MC & 0.189 \\
Normal & $2(14.3)$ & $0(0)$ & $2(40)$ & MC & 0.169 \\
MC Monte Carlo test & & & & \\
\hline
\end{tabular}

Table (4) Relation between findings on carotid Doppler and EDV among the studied patients:

\begin{tabular}{llllll}
\hline EDV & \multicolumn{1}{c}{$\begin{array}{c}\text { Carotid doppler } \\
\text { Atherosclerosis }\end{array}$} & $\begin{array}{l}\text { Atherosclerosis with plaques } \\
\mathrm{N}=31(\%)\end{array}$ & $\begin{array}{l}\text { Normal } \\
\mathrm{N}=5(\%)\end{array}$ & \multirow{2}{*}{$\chi^{2}$} & $\mathrm{p}$ \\
& $\mathrm{N}=14(\%)$ & $4(12.9)$ & $0(0)$ & $\mathrm{MC}$ & 0.651 \\
\hline High & $1(7.1)$ & $27(87.1)$ & $5(100)$ & & \\
\hline Normal & $13(92.9)$ & &
\end{tabular}

MC Monte Carlo test

Table (5) Relation between findings on carotid Doppler and PSV among the studied patients:

\begin{tabular}{llllll}
\hline PSV & $\begin{array}{l}\text { Carotid doppler } \\
\text { Atherosclerosis }\end{array}$ & $\begin{array}{l}\text { Atherosclerosis with plaques } \\
\mathrm{N}=31(\%)\end{array}$ & $\begin{array}{l}\text { Normal } \\
\mathrm{N}=5(\%)\end{array}$ & $\chi^{2}$ & $\mathrm{p}$ \\
\hline High & $9(64.3)$ & $17(54.8)$ & $0(0)$ & $\mathrm{MC}$ & $0.04^{*}$ \\
Normal & $5(35.7)$ & $14(45.2)$ & $5(100)$ & & \\
\hline
\end{tabular}

MC Monte Carlo test ${ }^{*} \mathrm{p}<0.05$ is statistically significant

Table (6) Performance of carotid Doppler in prediction of silent strokes among the studied patients:

\begin{tabular}{llll}
\hline Abnormal findings on carotid Doppler & $\begin{array}{l}\text { Silent stroke } \\
\text { Present }\end{array}$ & Absent & Total \\
\hline Present & 43 & 2 & 45 \\
Absent & 3 & 2 & 5 \\
Total & 46 & 4 & 50 \\
$\mathbf{9 3 . 5 \%}$ & $50 \%$ & $95.6 \%$ & $40 \%$ \\
$*$ p $<0.05$ is statistically significant & & &
\end{tabular}

Table (7) Performance of carotid Doppler in prediction of high EDV among the studied patients:

\begin{tabular}{llll}
\hline Abnormal findings on carotid Doppler & EDV & Total \\
& High & Normal & \\
\hline Present & 5 & 40 & 45 \\
Absent & 0 & 5 & 5 \\
Total & 5 & 45 & 50 \\
$\mathbf{1 0 0 \%}$ & $11.1 \%$ & $11.1 \%$ & $100 \%$ \\
\hline
\end{tabular}

$* \mathrm{p}<0.05$ is statistically significant 
Benha medical journal, vol.39, special issue (radiology), 2022

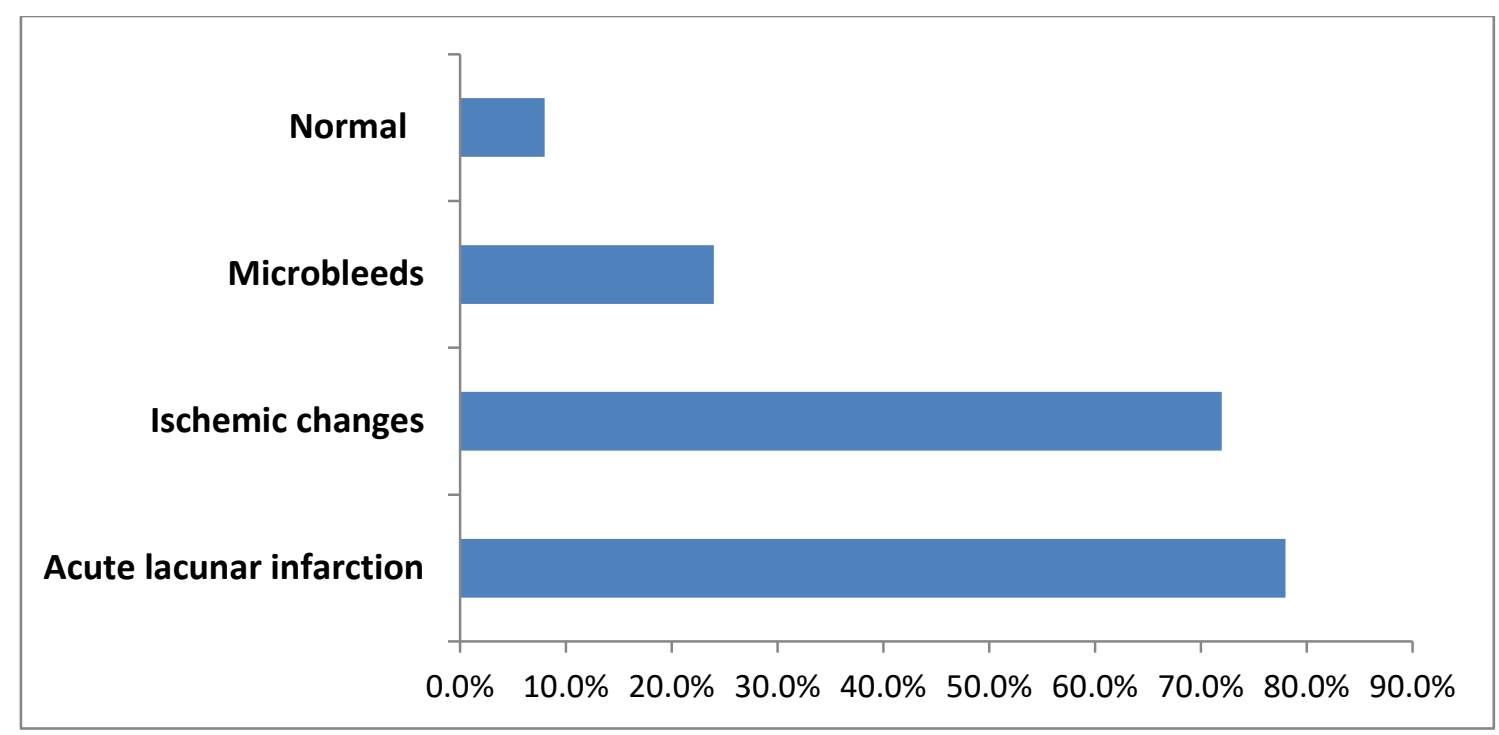

Figure (1) Simple bar chart showing distribution of the studied patients according to MRI findings

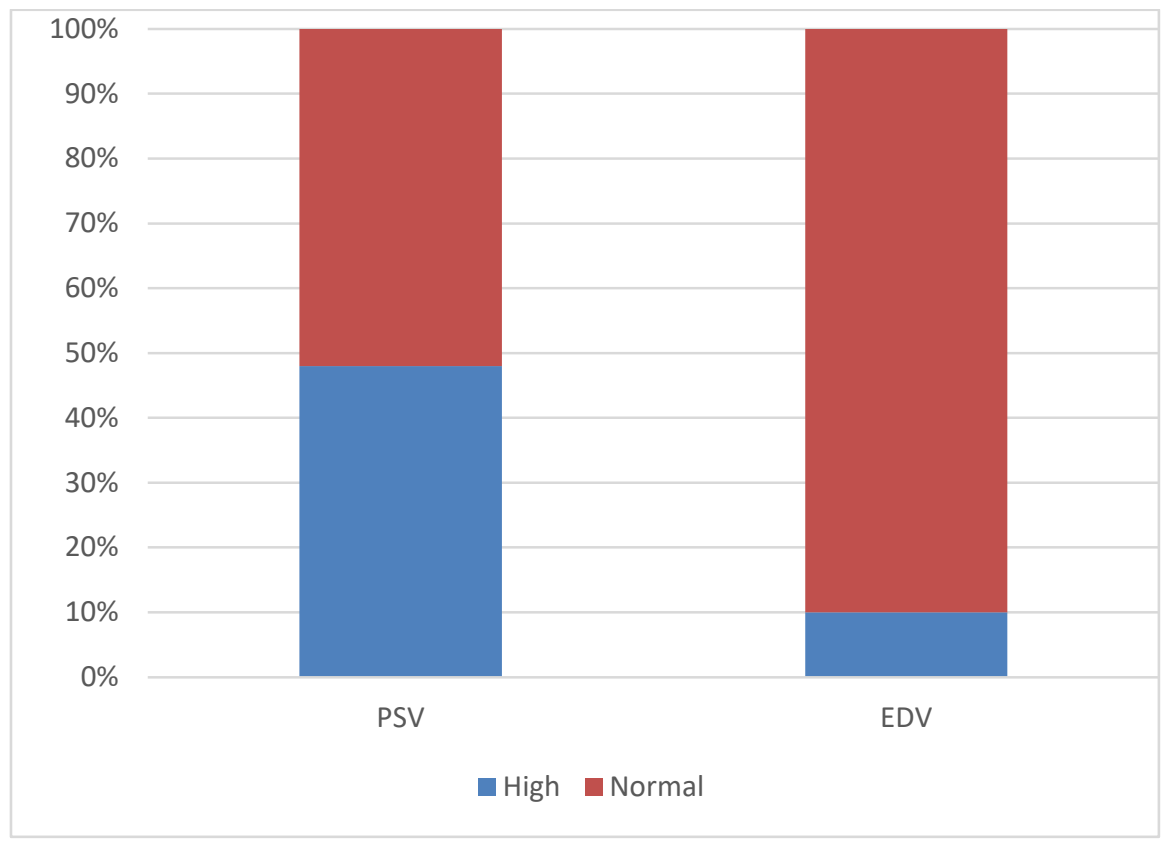

Figure (2) Compound bar chart showing distribution of the studied patients according to MRI findings 


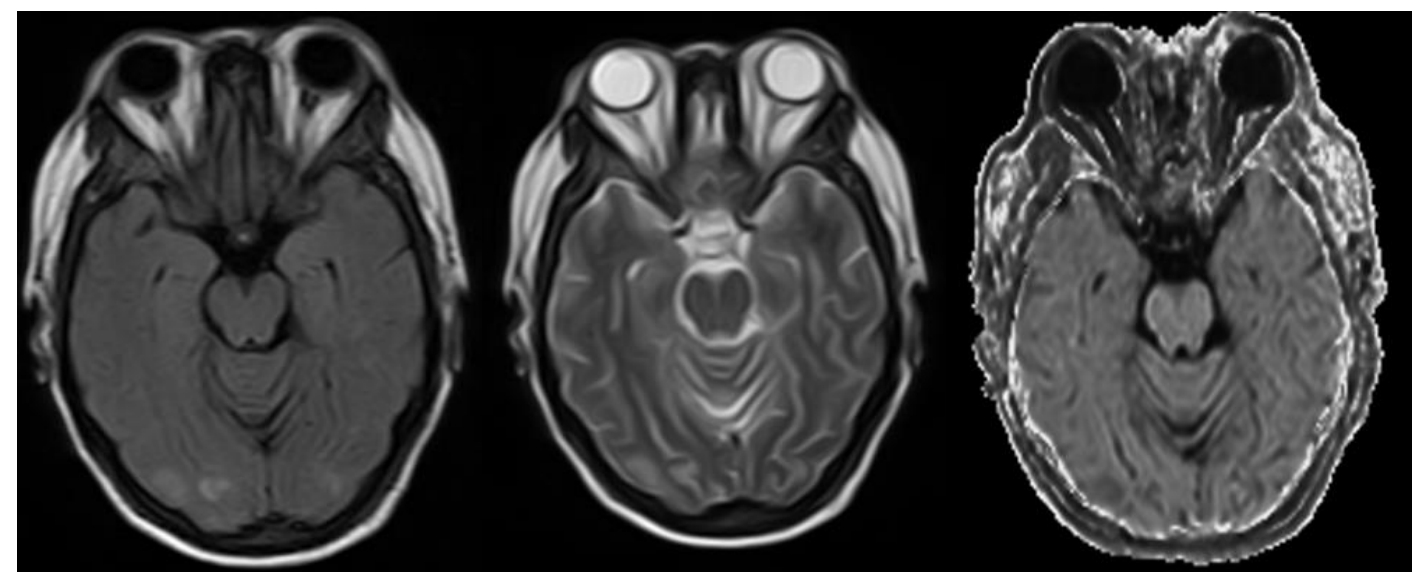

Figure 3:

MRI brain: Few foci of abnormal SI in both occipital regions display low signal in T1WI, high signal in T2WI and FLAIR images. Old lacunar infarcts.

B-Mode: Evidence of bilateral diffuse atherosclerotic changes in the extracranial carotid artery walls in the form of thickening of the intima-media complex $1.2 \mathrm{~mm}$. An adherent atheromatous plaque at the right common carotid artery. It is echogenic, homogenous, having regular surface measure $12 \times 2 \mathrm{~mm}$.

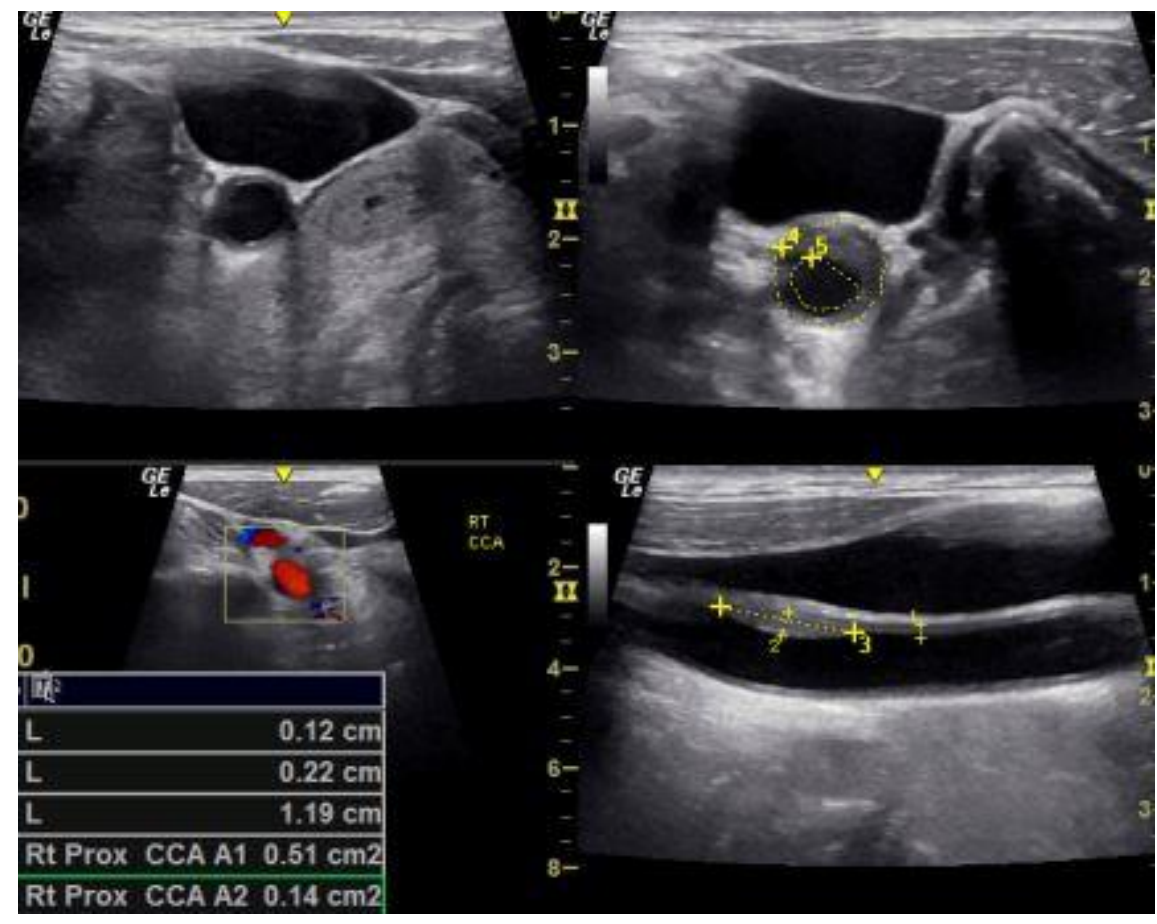

Figure 4 


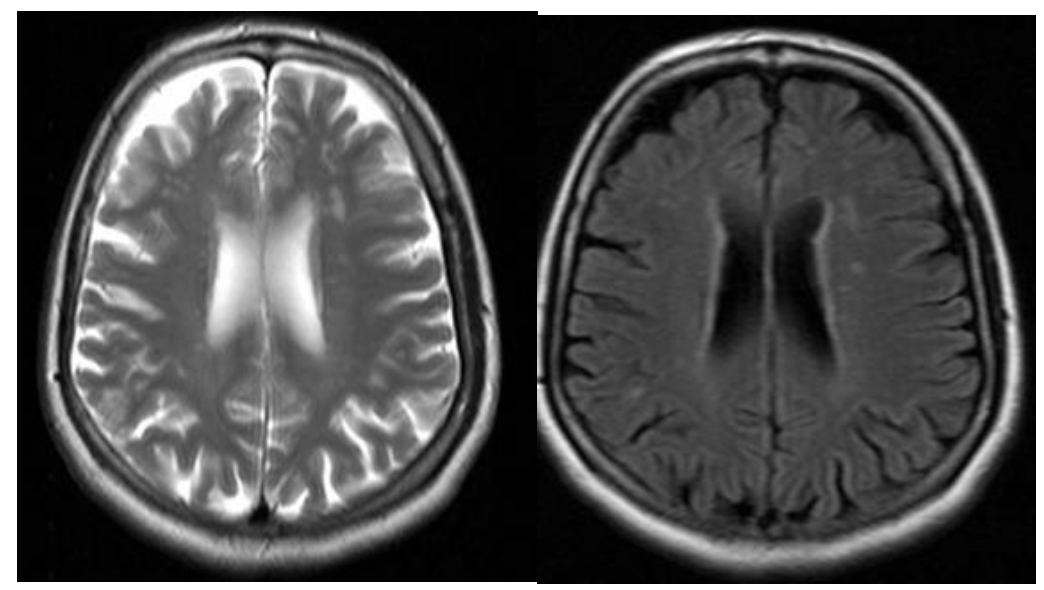

\section{Figure 5:}

MRI brain: Multiple periventricular lacunar infarctions at both cerebral hemispheres in T2 and FLAIR axial images.

B-Mode: Evidence of bilateral diffuse atherosclerotic changes in the extracranial carotid artery walls in the form of thickening of the intima-media complex $1.1 \mathrm{~mm}$.

Spectral analysis:

- Elevated PSV reaching $121 \mathrm{~cm} / \mathrm{sec}$.

- $\quad \mathrm{EDV}=29 \mathrm{~cm} / \mathrm{sec}$

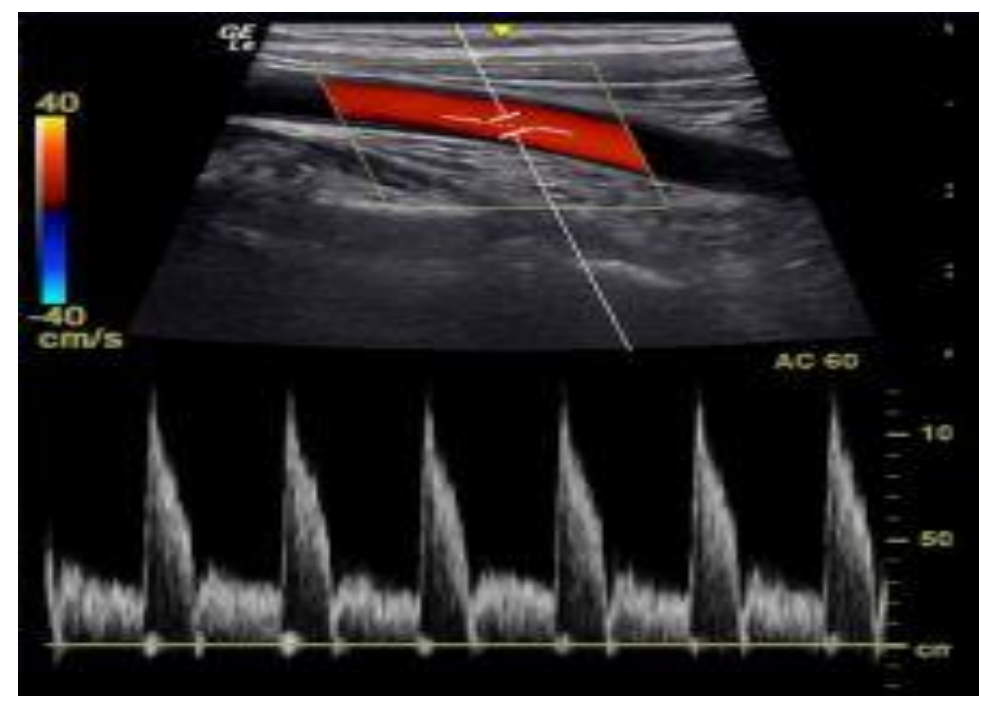

Figure 6

\section{Discussion}

In our study, IMT was measured at the common carotid bulb. We used ultrasoundbased measures of extracranial plaque and stenosis, measuring stenosis using the Doppler peak flow velocity during ultrasonography as we find that there is 
statistically significant association between PSV and findings on carotid Doppler among the studied patients. In our study, the frequency of a moderate- or high-grade carotid stenosis was very low.

Firstly, elderly with late onset hypertension, with increasing SBP values over time had slightly higher PSV, mainly on the left side and a clear relationship between high PSV in carotids, the grade of systolic blood pressure increase and $\mathrm{CBF}$ defects of arteriosclerotic origin probably due to dynamic effect of high pulse pressure, which simultaneously affects cerebral circulation. Secondly, subjects with early onset hypertension, with blood pressure decline over time, either due to dysautonomia or heart failure, where lower blood pressure express higher vascular lifetime-load, and where higher peripheral resistance in cerebral vessels and lower cardiac output could blur the pulse pressure related PSV increase and its effect on CBF (5).

In our study, we included 50 patients, all the patients were hypertensives, $40 \%$ of them were males. Age ranged from 50 to 74 years with mean 60.72 years.

Normal MRI findings occurred in only $8 \%$ of patients. Lacunar infarction, ischemic changes and microbleeds occurred in $78 \%$, $72 \%$ and $24 \%$ of patients.

Normal carotid findings occurred in only $10 \%$ of patients. Atherosclerosis without plaques was detected in $30 \%$ of patients while $60 \%$ of patients had combined atherosclerosis with plaques.

High PSV occurred in $48 \%$ of the studied patients.

There is significant agreement between presence of abnormal findings on carotid Doppler and findings of silent stroke by MRI.

Although there is non-significant association between carotid Doppler findings and each of MRI findings including lacunar infarction, ischemic changes, microbleeds or normal findings among the studied patients, There is statistically significant association between PSV and findings suggestive of microbleeds, lacunar infarction or normal appearance on MRI.

Abnormal carotid Doppler findings detect 43 out of 46 patients with findings suggestive of silent stroke with sensitivity 93.5\% and normal carotid Doppler occurred in 2 out of 4 patients with normal MRI study with specificity $50 \%$. Positive predictive value (PPV) was $95.6 \%$ while negative 
predictive value (NPV) was $40 \%$ and accuracy $90 \%$.

In accordance with our study, a study (6) examined the relationship between carotid atherosclerosis and the presence of SBI at brain MRI . They demonstrate a significant association between carotid atherosclerosis and the presence of SBI, with a pooled OR of 1.89 (95\% CI: 1.46-2.45, p< 0.0001).

Other researchers (7) assessed the association between carotid atherosclerosis and total WMH volume in a cohort of 94 subjects with a mean age of 71 years. At multivariable analysis, they found a significant association between age and WMH, but not between carotid stenosis and WMH.

A single, prospective study (8) reported an increased risk $(\mathrm{OR}=1.76)$ for severe $\mathrm{WMH}$ at 4 years of follow up in patients with carotid atherosclerosis.

\section{Conclusion}

Carotid atherosclerosis can be proposed as a marker for susceptibility to ischemic cerebral damage.

\section{References}

1. Fuchs FD, Whelton PK. High blood pressure and cardiovascular disease. Hypertension. 2020;75(2):285-92.

2. Riba-Llena I, Jarca CI, Mundet X, Tovar JL, Orfila F, López-Rueda A, et al. Investigating silent strokes in hypertensives: a magnetic resonance imaging study (ISSYS): rationale and protocol design. BMC Neurol. 2013;13(1):1-8.

3. e Fernandes JF, Pedro LM, Gonçalves I. The conundrum of asymptomatic carotid stenosisdeterminants of decision and evidence. Ann Transl Med. 2020;8(19).

4. Byrnes KR, Ross CB. The current role of carotid duplex ultrasonography in the management of carotid atherosclerosis: foundations and advances. Int J Vasc Med. 2012;2012.

5. Siennicki-Lantz A, Elmståhl S. Phenomenon of declining blood pressure in elderly-high systolic levels are undervalued with Korotkoff method. BMC Geriatr. 2011;11(1):1-9.

6. Moroni F, Ammirati E, Magnoni M, D’Ascenzo F, Anselmino M, Anzalone $\mathrm{N}$, et al. Carotid atherosclerosis, silent ischemic brain damage and brain atrophy: a systematic review and metaanalysis. Int J Cardiol. 2016;223:681-7.

7. Landi D, Maggio P, Lupoi D, Palazzo P, Altamura C, Falato E, et al. Cortical ischemic lesion burden measured by DIR is related to carotid artery disease severity. Cerebrovasc Dis. 2015;39(1):23-30.

8. Pico F, Dufouil C, Lévy C, Besançon V, de Kersaint-Gilly A, Bonithon-Kopp C, et al. Longitudinal study of carotid atherosclerosis and white matter hyperintensities: the EVA-MRI cohort. Cerebrovasc Dis. 2002;14(2):109-15.

To cite this article: Hesham M. Farouk, Osama T. Galal, Nada N. Ali. Carotid Doppler as a Screening Tool for Silent Cerebral Stroke in Hypertensive Patients. BMFJ 2022; 39 (Radiology):255-266. DOI: 10.21608/bmfj.2021.93559.1473 